\title{
Correlation of left atrial strain with left ventricular end-diastolic pressure in patients with normal left ventricular ejection fraction
}

\author{
Jia-Li Fan ${ }^{1} \cdot$ Bo Su${ }^{1} \cdot$ Xin Zhao ${ }^{1} \cdot$ Bing-Yuan Zhou ${ }^{1} \cdot$ Chang-Sheng Ma ${ }^{1}$ Hai-Peng Wang ${ }^{1} \cdot$ Sheng-Da Hu ${ }^{1}$. \\ Ya-Feng Zhou ${ }^{1} \cdot$ Yi-Jiao Ju ${ }^{1} \cdot$ Ming-Han Wang ${ }^{1}$
}

Received: 19 December 2019 / Accepted: 24 April 2020 / Published online: 3 May 2020

(C) The Author(s) 2020

\begin{abstract}
Left ventricular diastolic dysfunction (LVDD) remains challenging to be assessed by echocardiography. We sought to explore the relationship between left atrial strain and left ventricular (LV) diastolic function in patients with normal left ventricular ejection fraction (LVEF) by invasive left-heart catheterization. 55 consecutive individuals with LVEF $>50 \%$ underwent LV catheterization. Standard transthoracic echocardiography was performed during $12 \mathrm{~h}$ before or after the procedure. Left atrial (LA) strain were obtained by speckle tracking echocardiography. When LVEF $\geq 50 \%$, the group with elevated left ventricular end-diastolic pressure (LVEDP) $(\mathrm{n}=35)$ showed decreased left atrial reservoir strain (LASr) $(35.2 \pm 7.7 \%$ vs $21.3 \pm 7.2 \%$, $\mathrm{p}<0.001)$, left atrial conduit strain (LASct) $(17.6 \pm 6.3 \%$ vs $11.9 \pm 4.1 \%, \mathrm{p}<0.001)$, left atrial contraction strain (LAScd) $(16.6 \pm 7.2 \%$ vs $9.5 \pm 5.0 \%, \mathrm{p}<0.001)$ and increased $\mathrm{E} / \mathrm{e}^{\prime}$ ration $(8.9 \pm 2.6$ vs $10.1 \pm 3.5, \mathrm{p}=0.17)$. LVEDP negatively correlated with $\operatorname{LASr}(\mathrm{R}=0.662, \mathrm{p}<0.001)$, LASct $(\mathrm{R}=0.575, \mathrm{p}<0.001)$ and $\operatorname{LAScd}(\mathrm{R}=0.456, \mathrm{p}<0.001)$, but not with $\mathrm{E} / \mathrm{e}^{\prime}$. LASr, LASct and LAScd were all independent predictors of elevated LVEDP ( $\mathrm{p}<0.05)$, with a higher C-statistic for the model including LASr $(0.95,0.86$ and 0.93 respectively). The area under the curve (AUC) for LASr is 0.914 (cutoff value is $26.7 \%$, sensitivity is $90 \%$, specificity is $82.9 \%$ ). In patients with normal LV ejection fraction, left atrial strain presented good correlation with LVEDP, and LASr was superior to LASct and LAScd to predict LVEDP. LA strain demonstrated better agreement with the invasive reference than E/e'.
\end{abstract}

Keywords Left ventricular diastolic dysfunction $\cdot$ Left atrial strain $\cdot \mathrm{HFpEF}$

\section{Introduction}

Left ventricular diastolic dysfunction is an independent predictor of all-cause mortality in the general population, even in the preclinical stage [1], and evidence of LVDD is required for the diagnosis of heart failure with preserved ejection fraction (HFpEF) [2,3]. So, it is increasingly important to evaluate LVDD accurately in routine clinical practice. Elevated left ventricular (LV) filling pressures are the main physiologic consequence of LV diastolic dysfunction [4].

First authors: Jia-Li Fan and Bo Su.

Xin Zhao

zxdemail@126.com

Bing-Yuan Zhou

zhoubinyuan@sina.com

1 The First Affiliated Hospital of Soochow University, Suzhou, China
Although invasive methods are considered the "gold standard" for evaluating left ventricular filling pressures and LV diastolic function, echocardiography is routinely used as a noninvasive alternative.

The 2016 ASE/SCAI guidelines streamline the use of four variables into a single algorithm to assess LV diastolic function, while accuracy will be affected in the presence of pulmonary arterial hypertension, severe tricuspid valve lesions, and low right atrial and right ventricular filling pressure etc. [4]. An accurate assessment of left ventricular diastolic function by transthoracic echocardiography is still needed. Speckle tracking is applied to directly reflect the intrinsic deformation of left atrium, which has a relatively independent load environment and geometric model and is less affected by the load change [5, 6]. It is suggested that left atrial strain should be used in diagnosis of LVDD [7]. The previous study indicated that LA strain correlates well with LVEDP, as well as pulmonary capillary wedge pressure [7-10]. While LASct and LAScd are rarely mentioned in 
previous studies, cut-off value for LA strain is still undefined by invasive gold reference. Our study intended to explore the correlation between LA strain and LV diastolic dysfunction in patients with normal LVEF.

\section{Methods}

\section{Population}

Between June 2018 and November 2019, we prospectively studied 55 patients with LVEF $\geq 50 \%$ (mean age, 63 years old, 38 men [69.1\%]) referred for left heart catherization, and echocardiography was completed within $12 \mathrm{~h}$ before or after catheterization. Patients with confirmed or suspected coronary artery disease underwent left heart catheterization. Those with Atrial fibrillation, ST-segment elevation myocardial infarction (STEMI), non-ST-segment elevation myocardial infarction (NSTEMI), moderate or greater tricuspid regurgitation, moderate or greater mitral regurgitation, any mitral or aortic stenosis, prosthetic valves, hemodynamic instability or poor echocardiography imaging were excluded. The study was approved by the institutional enrolling board of the first affiliated hospital of Soochow university.

\section{Echocardiography}

Echocardiographic measurements were performed by two board-certified echocardiographers blinded to the LVEDP who finalized each measurement by consensus. The twodimensional echocardiographic imaging of all subjects was performed by GE Vivid E9 and GE Vivid E95 equipment (Norway) $2.5 \mathrm{MHz}$ transducer. The LA dimension was measured in the parasternal long-axis view at the ventricular end-systole. Mitral diastolic inflow was interrogated using pulsed-wave Doppler from the apical four-chamber view at the level of the mitral leaflet tips. Mitral early diastolic peak (E-wave) and late peak (A-wave) velocities and E/A ratio were measured. In the apical two and four-chamber views including the entire left atrium, LAV was determined using biplane Simpson method at end-systolic frames just before mitral valve opening (maximal left atrial volume, LAVmax), at end-diastolic frames coincided with the R-wave on the electrocardiogram (minimal left atrial volume, LAVmin) and during mid-diastole phase which is before electrocardiographic P-wave (left atrial volume before A wave, LAVpre-A). Values were then indexed to the body surface area (LAVimax, LAVimin and LAVIpre-A). left atrial ejection fraction (LAEF) were calculated by LAVImax minus LAVImin. In addition, the septal mitral annulus early ( $\left.\mathrm{E}^{\prime}\right)$ velocity was measured by tissue Doppler imaging, and the $\mathrm{E} / \mathrm{E}^{\prime}$ ratio was calculated using a cutoff value $>15$ to represent elevated LV filling pressure. LVEF was measured using
Simpson's method, which was used as a standard index of global LV systolic function. All echocardiographic measurements used in the analysis were averaged from 3 heart beats.

\section{LA longitudinal strain analysis}

The two-dimension strain analysis package available on the Echo PAC work station (GE Healthcare) was used to measure the LA strain and strain rate. Similar to the left ventricle, the complete myocardial region of interest (ROI) of the LA is defined by the endocardial border. An adjustable ROI with a default width of $3 \mathrm{~mm}$ is recommended. The user can adjust the size and shape to include the thickness of LA wall and to avoid including the pericardium. The software divided the atrial endocardium into six segments, poorly displayed segments were automatically rejected by the software and excluded from the analysis. The global longitudinal strain and strain rate curves were generated by the software for each apical view. The operator could repeat the imaging or change software parameters such as the width of the region of interest and the smoothing functions to obtain satisfactory tracking. Apical four and two-chamber views were obtained in three consecutive heart beats, and electrocardiograph was recorded in the meantime. The LA strain is defined as the absolute strain value in LA three phases, which includes reservoir strain in systole (LASr), conduit strain in early diastole (LASct) and contraction strain in late diastole (LAScd). LV end-diastole was defined as initial zero reference point. The respective strains are LASr, calculated as difference between onset of filling and end-diastole; LAScd, calculated as difference between onset of atrial contraction (before start of Doppler A-wave) and onset of filling; LASct, calculated as difference between end-diastole and onset of atrial filling. The global strain was calculated as the average of both respective values form both views. (Fig. 1).

\section{Invasive LV pressure measurements}

A $6 \mathrm{~F}$ pigtail catheter was placed in the left ventricle to obtain the invasive LV pressure. Excluding the unstable baseline of LV filling pressure, LVEDP was measured by mean values of 3-5 cardiac cycles. Image $J$ was used to measure LVEDP at the beginning of QRS on Electrocardiography. A LVEDP value $>16 \mathrm{mmHg}$ was defined as elevated LV filling pressure $[3,11]$. LVEDP values were measured by two investigators blinded to echocardiographic data.

\section{Statistical analysis}

Analyses were performed using the SPSS 25.0. Continuous variables which are normally distributed are presented as mean $\pm \mathrm{SD}$. Variables that are not normally distributed 




Fig. 1 LA two-dimensional strain. Left atrial strain during reservoir phase (LASr) is calculated as difference between onset of filling and end-diastole; Left atrial strain conduit phase (LAScd), calculated as

will be presented as median with inter-quartile ranges (IQR $=25$ th-75th percentile). T test and Mann-Whitney $\mathrm{U}$ test was used for comparison of groups. Categorical variables are expressed as absolute numbers and respective percentages, categorical variables were compared with chi squared tests. LA strain and LV diastolic dysfunction parameters such as septal $\mathrm{e}^{\prime}$ velocity, lateral $\mathrm{e}^{\prime}$ velocity, E/e', E/A, LAEF, tricuspid regurgitation velocity, LAVI and LVEDP were evaluated using univariate logistic regression analysis. Differences with $\mathrm{p}$ values less than 0.05 were considered significant. The independent predictive value of echocardiographic variables was evaluated in three different multivariate model. Predictors of elevated LVEDP with $p$ value $\leq 0.12$ in the univariate analysis and age, were included in multivariate models. In our three models, LASr LASct and LAScd were analyzed separately due to their multicollinearity, with other control variables the same. The $\mathrm{C}$-statistic was calculated in each model, in order to allow comparison between them. Receiver operating characteristics (ROC) curves were created to compare difference between onset of atrial contraction and onset of filling; Left atrial strain contraction phase (LASct), calculated as difference between end-diastole and onset of atrial filling

the performance of multiple variables in determining increased LVEDP.

\section{Results}

\section{Characteristics of population}

Patients' clinical characteristics and echocardiographic measurements are shown in Table 1. LVEDP was elevated in 35 patients $(63.6 \%)$, and normal in $20(36.4 \%)$ patients. The study population $(n=55)$ has a mean age of $63 \pm 10.0$ years, among which 17 (31\%) were female. Most characteristics are similar in both groups, such as age, diabetes, CAD etc. Significant differences between the two groups were observed in LA strain (LASr, LASct, LAScd). Vital signs were not significantly different when echocardiography and catheterization were performed. 
Table 1 Characteristics of patients in two groups defined by LVEDP

\begin{tabular}{|c|c|c|c|}
\hline Variable & LVEDP $\leq 16$ mmHg $(\mathrm{n}=20)$ & $\begin{array}{l}\text { LVEDP }>16 \mathrm{mmHg} \\
(\mathrm{n}=35)\end{array}$ & $\mathrm{p}$ \\
\hline Male & $15(75 \%)$ & $23(66 \%)$ & 0.48 \\
\hline Age & $63.8 \pm 8$ & $62.4 \pm 11.0$ & 0.63 \\
\hline BMI & $24.1 \pm 3.1$ & $24.5 \pm 3$ & 0.83 \\
\hline HR (beats/min) & $63.6 \pm 10$ & $68.1 \pm 9$ & 0.10 \\
\hline \multicolumn{4}{|l|}{ Medical history } \\
\hline HTN & $12(60 \%)$ & $29(83 \%)$ & 0.06 \\
\hline Diabetes & $4(20 \%)$ & $10(29 \%)$ & 0.50 \\
\hline CAD & $9(45 \%)$ & $11(31 \%)$ & 0.32 \\
\hline COPD & $1(5 \%)$ & $1(3 \%)$ & 0.69 \\
\hline CKD(stage $\geq 3$ )or ESRD & $1(5 \%)$ & $1(3 \%)$ & 0.69 \\
\hline \multicolumn{4}{|c|}{ Medications before catheterization } \\
\hline Diuretic & 2 & 3 & 1.00 \\
\hline Calcium blocker & 5 & 15 & 0.19 \\
\hline BB & 6 & 17 & 0.18 \\
\hline ACEI & 5 & 8 & 0.86 \\
\hline ARB & 2 & 3 & 1.00 \\
\hline \multicolumn{4}{|l|}{ Angiographic findings } \\
\hline \multicolumn{4}{|l|}{ Stenosis } \\
\hline None significant stenosis & 9 & 21 & 0.28 \\
\hline$<50 \%$ of diameter & 3 & 5 & 1.00 \\
\hline$>50 \%$ of diameter & 6 & 4 & 0.18 \\
\hline Total occlusion & 2 & 5 & 0.97 \\
\hline \multicolumn{4}{|c|}{ Numbers of vessels with significant stenosis $>50 \%$} \\
\hline 1 & 4 & 6 & 1.00 \\
\hline 2 & 3 & 1 & 0.26 \\
\hline 3 & 1 & 2 & 1.00 \\
\hline \multicolumn{4}{|l|}{ Vessels with stenosis $>50 \%$} \\
\hline LM & 1 & 1 & 1.00 \\
\hline LAD & 7 & 7 & 0.34 \\
\hline LCX & 3 & 1 & 0.26 \\
\hline RCA & 1 & 3 & 1.00 \\
\hline \multicolumn{4}{|l|}{ Echocardiographic parameters } \\
\hline Mitral E (cm/s) & $66.1 \pm 10.3$ & $68.8 \pm 14.7$ & 0.47 \\
\hline Mitral A (cm/s) & $76.8 \pm 17.8$ & $87.2 \pm 21.6$ & 0.07 \\
\hline E/A ratio & $0.8(0.7-0.8)$ & $0.8(0.6-0.9)$ & 0.19 \\
\hline Septal e' velocity $(\mathrm{cm} / \mathrm{s})$ & $6.7 \pm 2.0$ & $6.2 \pm 1.9$ & 0.356 \\
\hline Lateral e' velocity $(\mathrm{cm} / \mathrm{s})$ & $9.4 \pm 2.5$ & $8.6 \pm 3.1$ & 0.385 \\
\hline E/e' ratio & $8.5(6.9-10.8)$ & $9.9(8.1-11.1)$ & 0.24 \\
\hline TR peak velocity $(\mathrm{m} / \mathrm{s})$ & $2.3(2.1-2.5)$ & $2.3(2.1-2.6)$ & 0.85 \\
\hline LVEF (\%) & $66.7 \pm 8.9$ & $64.6 \pm 7.2$ & 0.95 \\
\hline LAVimax $\left(\mathrm{ml} / \mathrm{m}^{2}\right)$ & $26.9 \pm 9.5$ & $27.7 \pm 9.6$ & 0.89 \\
\hline $\operatorname{LAVimin}\left(\mathrm{ml} / \mathrm{m}^{2}\right)$ & $10.6(7.6-16.9)$ & $10.8(7.7-13.2)$ & 1.00 \\
\hline LAVi pre-A $\left(\mathrm{ml} / \mathrm{m}^{2}\right)$ & $17.8 \pm 6.8$ & $19.8 \pm 6.7$ & 0.54 \\
\hline LAEF $(\%)$ & $54.5 \pm 8.1$ & $55.3 \pm 10.3$ & 0.772 \\
\hline $\operatorname{LASr}(\%)$ & $35.2 \pm 7.7$ & $21.3 \pm 7.2$ & $<0.001$ \\
\hline LASct (\%) & $17.6 \pm 6.3$ & $11.9 \pm 4.1$ & $<0.001$ \\
\hline LAScd (\%) & $14.7(12.2-19.7)$ & $9.6(5.8-12.4)$ & $<0.001$ \\
\hline
\end{tabular}

Data are expressed as numbers or as mean \pm SD. See text for details

$L V E D P$ left ventricular end-diastolic pressure, $H R$ heart rate, $C A D$ coronary artery disease, $C K D$ chronic kidney disease, $C O P D$ chronic obstructive pulmonary disease, ESRD end-stage renal disease, $H T N$ hypertension. $B B \beta$-blocker, $A C E I$ angiotensin-converting enzyme, $A R B$ angiotensin II receptor blocker, $L M$ left main coronary artery, $L A D$ left anterior descending coronary artery, $L C X$ left circumflex coronary artery, $R C A$ right coronary artery, $T R$ tricuspid regurgitation, $L V E F$ left ventricular ejection fraction, $L A V i$ left atrium volume index, $L A E F$ left atrial ejection fraction, $L A S r$ LA reservoir strain, $L A S c t$ LA conduit strain, LAScd LA contraction strain 
Table 2 Multivariate regression analysis to identify predictors for elevated LVEDP

\begin{tabular}{|c|c|c|c|c|c|c|}
\hline \multirow[t]{2}{*}{ Model } & \multirow[t]{2}{*}{ Variables } & \multicolumn{2}{|l|}{ Univariate analysis } & \multicolumn{2}{|c|}{ Multivariate analysis } & \multirow[t]{2}{*}{ C-statistic } \\
\hline & & OR $(95 \% \mathrm{CI})$ & $\mathrm{p}$ & OR $(95 \%$ CI $)$ & $\mathrm{p}$ & \\
\hline \multirow[t]{5}{*}{1} & LASr & $0.76(0.67-0.89)$ & $<0.001$ & $0.75(0.64-0.88)$ & 0.001 & \multirow[t]{5}{*}{0.95} \\
\hline & Age & $0.98(0.92-1.04)$ & 0.42 & $0.93(0.81-1.06)$ & 0.29 & \\
\hline & Mitral A & $1.02(1.00-1.06)$ & 0.12 & $1.06(0.99-1.13)$ & 0.12 & \\
\hline & HR & $1.07(1.00-1.14)$ & 0.06 & $1.04(0.93-1.17)$ & 0.51 & \\
\hline & HTN & $0.36(0.10-1.27)$ & 0.11 & $0.22(0.03-1.73)$ & 0.15 & \\
\hline \multirow[t]{5}{*}{2} & LASct & $0.78(0.65-0.92)$ & 0.003 & $0.72(0.57-0.90)$ & 0.004 & \multirow[t]{5}{*}{0.86} \\
\hline & Age & $0.98(0.92-1.04)$ & 0.42 & $0.95(0.86-1.04)$ & 0.27 & \\
\hline & Mitral A & $1.02(1.00-1.06)$ & 0.12 & $1.05(0.99-1.11)$ & 0.09 & \\
\hline & HR & $1.07(1.00-1.14)$ & 0.06 & $1.04(0.95-1.14)$ & 0.42 & \\
\hline & HTN & $0.36(0.10-1.27)$ & 0.11 & $0.18(0.04-0.92)$ & 0.04 & \\
\hline \multirow[t]{5}{*}{3} & LAScd & $0.76(0.64-0.90)$ & 0.001 & $0.71(0.57-0.89)$ & 0.003 & \multirow[t]{5}{*}{0.93} \\
\hline & Age & $0.98(0.92-1.04)$ & 0.42 & $0.93(0.81-1.06)$ & 0.27 & \\
\hline & Mitral A & $1.02(1.00-1.06)$ & 0.12 & $1.03(0.98-1.08)$ & 0.21 & \\
\hline & HR & 1.07 (1.00-1.14) & 0.06 & $1.08(0.98-1.20)$ & 0.11 & \\
\hline & HTN & $0.36(0.10-1.27)$ & 0.11 & $0.39(0.07-2.12)$ & 0.27 & \\
\hline
\end{tabular}

CI confidence interval, HR heart rate, LASr LA reservoir strain, LASct LA conduit strain, LAScd LA contraction strain, $H T N$ hypertension
Fig. 2 Scatter plots of variables with correlation to LVEDP, respectively LASr, LASct, LAScd and $\mathrm{E} / \mathrm{e}^{\prime}$ ratio
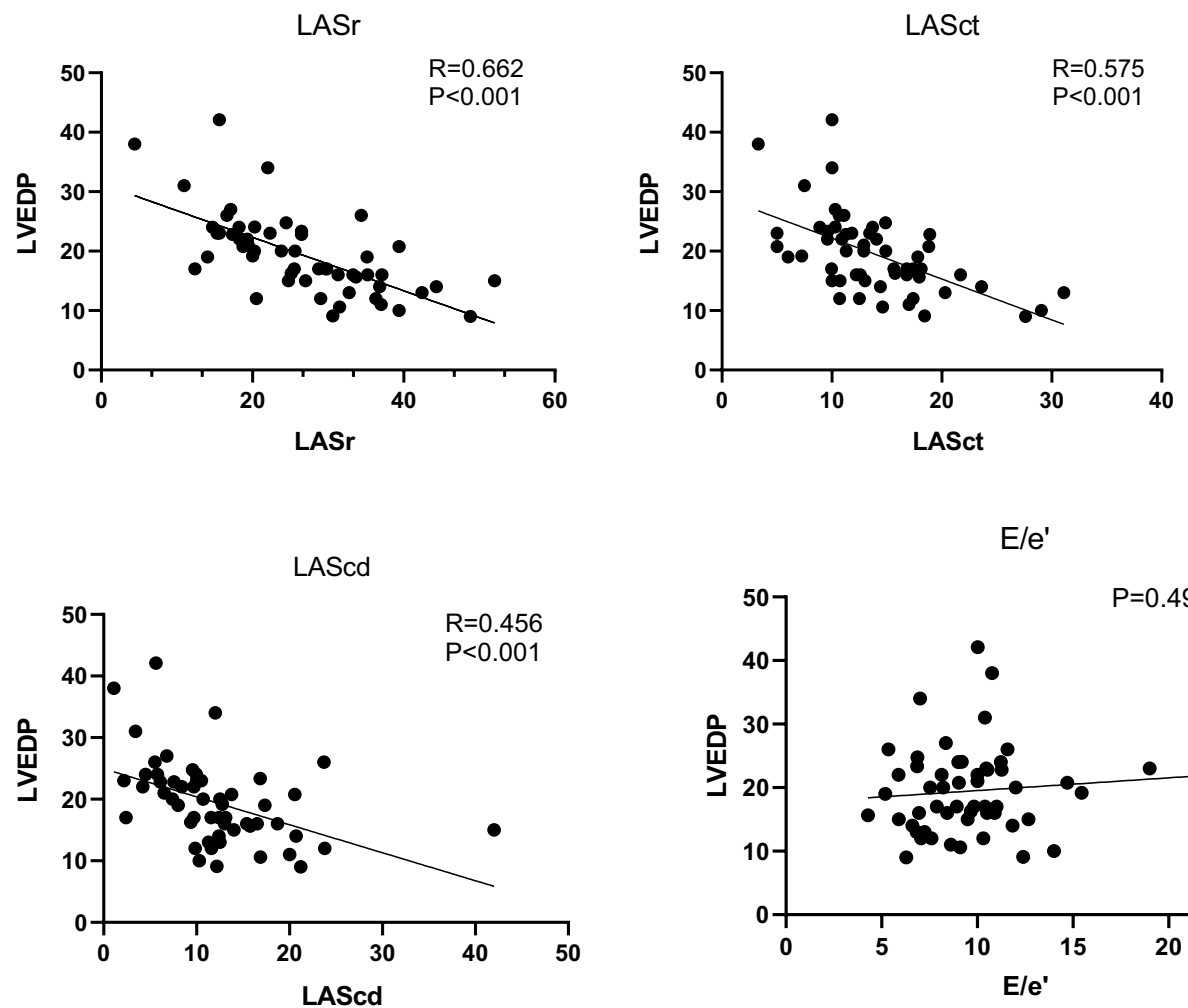

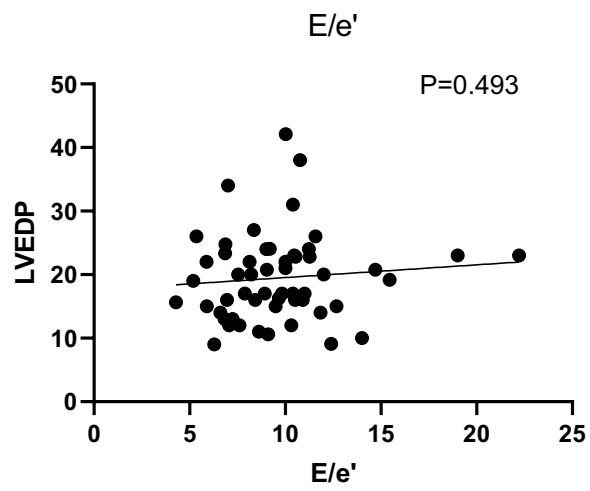

\section{Correlation between echocardiography assessment and LVEDP}

The LA strain and LVEDP are correlated negatively: LASr $(\mathrm{p}<0.001, \mathrm{R}=0.662)$, LASct $(\mathrm{p}<0.001, \mathrm{R}=0.575)$, LAScd
( $\mathrm{p}<0.001, \mathrm{R}=0.456)$. No significant correlation was found between the LVEDP and E/e' $(p=0.493)$. Figure 2 is scatter diagram of LA strain and E/e'. LASr has a stronger correlation with LVEDP, compared to other variables observed in our study. 
The predictive values of LASr, LASct and LAScd were evaluated in three different multivariate logistic regression analyses (Table 2), which contained the same variables (age, Mitral A, HR and hypertension), differing only by control variables. The LASr, LASct and LAScd were independent predictors for LVEDP $>16 \mathrm{mmHg}$ in their respective models. The model shows that LASr has a higher C-statistic when compared to the model with LASct and LAScd.

\section{The accuracy of LVEDP $>16 \mathrm{mmHg}$ predicted by LA strain}

Figure 3 shows that LA strain has good diagnostic accuracy for LVEDP > $16 \mathrm{mmHg}$. The area under the curve (AUC) for LASr is 0.914 (cutoff value is $26.7 \%$, sensitivity is $90 \%$, specificity is $82.9 \%$ ). For LASct, the AUC is 0.769 (cutoff value is $12.0 \%$, sensitivity is $85 \%$, specificity is $57 \%$ ). The
AUC of LAScd is 0.844 (cutoff value is $11.0 \%$, sensitivity is $90.0 \%$, specificity is $68.6 \%$ ), which shows that LASr can predict LVEDP better than LASct and LAScd.

\section{Discussion}

Left atrial strain presents significant feasibility and reproducibility, which has recently emerged as a powerful assessment in evaluation of left ventricular diastolic dysfunction [4]. We aim to evaluate the diagnostic accuracy of LA strain for LVDD in patients with normal LVEF by gold standard reference. In this study we demonstrated that left atrial strain was significantly impaired in the group with elevated LVEDP in patients with $\mathrm{LVEF} \geq 50 \%$. LASr showed a stronger correlation with LVEDP than with LASct, LAScd and other conventional diastolic echocardiography parameters. LA reservoir strain at cut-off of $26.7 \%$ predicted LVEDP $>16 \mathrm{mmHg}$
Fig. 3 The accuracy of LVEDP $>16 \mathrm{mmHg}$ predicted by LA strain and E/ e'. Receiver operating characteristic (ROC) curves of LASr, LASct and LAScd for prediction of LV filling pressure
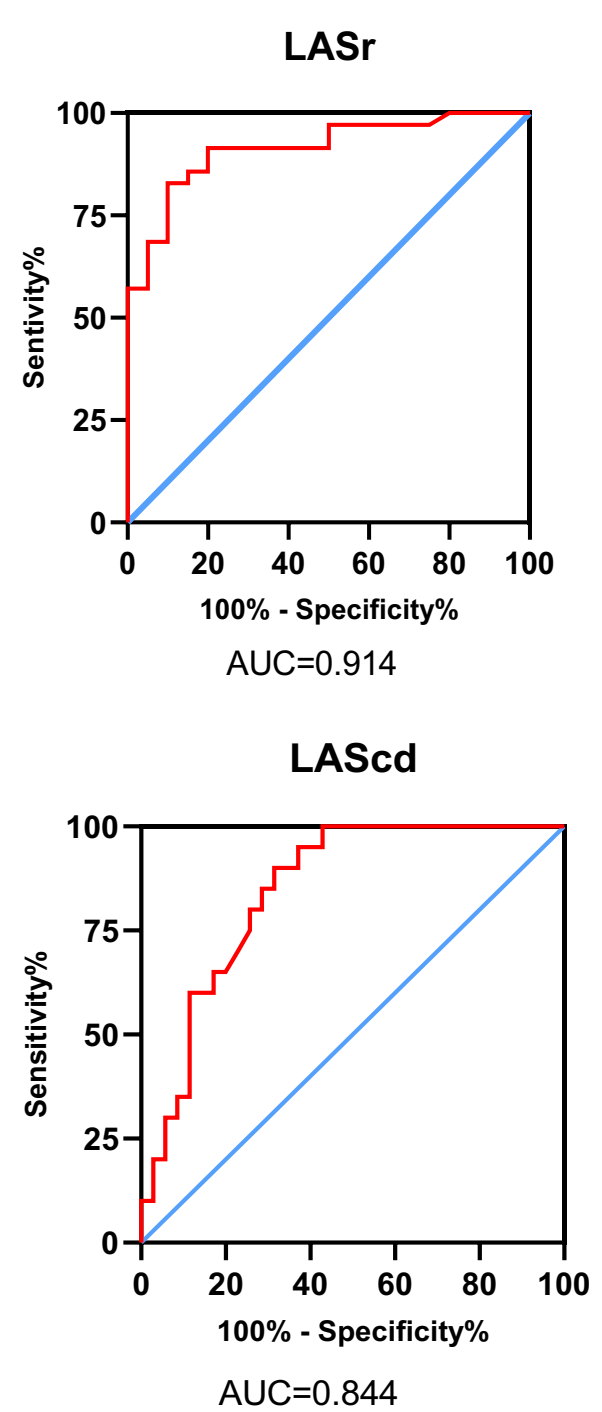
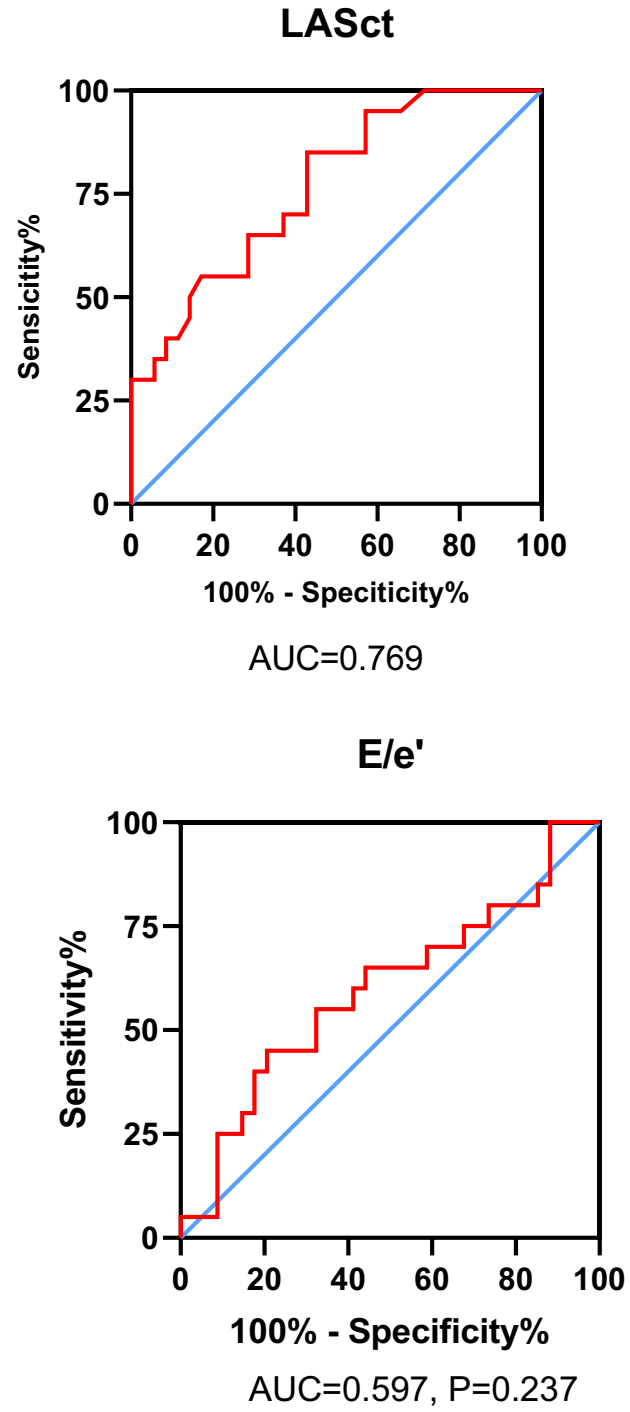
with $90 \%$ sensitivity and $82.9 \%$ specificity, which performed a higher diagnosis accuracy than LASct, LAScd and E/e'.

In the early phase of LVDD, reduced ventricular compliance and elevated LVEDP result in decreasing passive early transmitral diastolic flow, then atrial pump function enhances to compensate LV filling. As left ventricular distensibility declines further, atrial pressure rises to maintain cardiac output, which may blunt the compliance of the LA. LA function is impaired as a consequence of chronically elevated LVEDP and decreased LA compliance [12]. This may be the potential mechanism for the inverse correlation between LA strain and LVEDP.

Multiple recent studies have demonstrated the correlation between LA strain and hemodynamic parameters [8, 9], but for patients with $L V E F \geq 50 \%$, investigations with sufficient sample size about correlation between LA strain and LVEDP obtained by invasive gold standard reference, are still needed, and a definitive cut-off value for abnormal LA strain is still undefined.

Our study is consistent with the findings observed by Cameli and colleagues. They found that LASr was strongly negatively correlated with pulmonary capillary wedge pressure (PCWP) in patients with heart failure, which performed excellent sensitivity and specificity of $100 \%$ and $93 \%$ in assessing PCWP [9]. However, LVEDP $>16 \mathrm{mmHg}$ was defined as LVDD instead of PCWP $\geq 18 \mathrm{mmHg}$ in our study, which may recognize the patients at early stage of LVDD, because elevated PCWP only occurs at a decompensated LA when LVEDP increases significantly. Previous study showed that PCWP frequently underestimates LVEDP, and it only had a moderate ability to discriminate patients with normal or elevated LVEDP [13].

Amita Singh et al. have reported that LASr could predict elevated filling pressure with high accuracy and identified a cutoff value for LASr [8], while we have a lager sample size which may provide a more convincing evidence for cut off value of LA strain in patients with normal LVEF. Besides LASr, our study also demonstrated the correlation between LASct, LAScd and LVEDP. In our study LA strain presented a more sensitive diagnostic value for LVDD than conventional diastolic echocardiography assessments such as E/e', which indicates that LA strain can recognize patients with LVDD even at early stage.

Poor correlation between E/e' and LVEDP was observed in this study, which seems to contradict previous reports that E/e' correlated with PCWP or LVEDP significantly [9, $10,14,15]$. Possible explanations are as follows. Firstly, Previous study showed that E/e' between 8 and 15 predicts LV Filling Pressure with poor accuracy [16]. However, $34(61.8 \%)$ patients' $E / \mathrm{e}^{\prime}$ ratio is between 8 and 15 in our study. Secondly, the accuracy of E/e' ratio is uncertain in patients with advance decompensated HF, presence of cardiac resynchronization therapy, mitral annular calcification, surgical rings, prosthetic valves, several mitral regurgitations, atrioventricular or intraventricular conduction delay and symptomatic hypertrophic cardiomyopathy $[4,14,17]$.

Our study showed that LAVI correlated with LVEDP poorly. LA dilatation usually implies the chronic, long-term elevation of LA pressure, and LAV can be normal in the early phase of LVDD $[18,19]$. Many studies have suggested that LA strain may be superior to LAVi in evaluating LVDD even in the absence of LA enlargement $[5,6]$, which indicated that LA strain has better sensitivity. Moreover, LAV rarely normalizes with the reduction of LV filling pressure, and strong correlation of reduced LV filling pressure and improved LA strain demonstrates that LA strain may evaluate LA function remodeling better than LA size [20].

Current echocardiographic parameters show moderate sensitivity in evaluating LVDD, with the presence and severity of LVDD failing to be determined for a significant proportion of patients. LA strain measured by speckle tracking echocardiography can reclassify patients even in the early phase of LVDD. LA strain also has advantages when diagnosing LVDD in mitral prosthesis, mitral annular calcification, bundle branch block, post-cardiac surgery and AF. LA strain, with high reproductivity and feasibility, is suggested to be incorporated into routine evaluation of LVDD, especially for patients who are classified in the indeterminate LVDD group using the conventional recommended algorithm.

\section{Limitations}

The sample size in this study is limited, so selection bias still exists, however increasing the number of individuals will reduce the selection bias. The study was conducted on patients from only one medical center, which might impact the population selected and, consequently, the variables analyzed. Left atrium imaging is difficult to obtain and the image definition is poor in some cases, and some echocardiographic images have poor repeatability in left atrial strain.

\section{Conclusion}

For patients with normal LV ejection fraction, left atrial strain presented better correlation with LVEDP than E/e'. Moreover, LASr presented a better diagnostic value in predicting LVEDP than LASct and LAScd. It is suggested that left atrial strain should be used in diagnosis of LVDD.

Funding Supported by Jiangsu Provincial Medical Innovation Team. No. CXTDA2017009. 


\section{Compliance with ethical standards}

Conflict of interest The authors declare that they have no conflict of interests.

Open Access This article is licensed under a Creative Commons Attribution 4.0 International License, which permits use, sharing, adaptation, distribution and reproduction in any medium or format, as long as you give appropriate credit to the original author(s) and the source, provide a link to the Creative Commons licence, and indicate if changes were made. The images or other third party material in this article are included in the article's Creative Commons licence, unless indicated otherwise in a credit line to the material. If material is not included in the article's Creative Commons licence and your intended use is not permitted by statutory regulation or exceeds the permitted use, you will need to obtain permission directly from the copyright holder. To view a copy of this licence, visit http://creativecommons.org/licenses/by/4.0/.

\section{References}

1. Redfield MM, Jacobsen SJ, Burnett JC et al (2003) Burden of systolic and diastolic ventricular dysfunction in the community: appreciating the scope of the heart failure epidemic. JAMA 289(2):194-202

2. Shah KS, Xu H, Matsouaka RA et al (2017) Heart failure with preserved, borderline, and reduced ejection fraction: 5-year outcomes. J Am Coll Cardiol 70(20):2476-2486

3. Ponikowski P, Voors AA, Anker SD et al (2016) 2016 ESC Guidelines for the diagnosis and treatment of acute and chronic heart failure: The Task Force for the diagnosis and treatment of acute and chronic heart failure of the European Society of Cardiology (ESC). Developed with the special contribution of the Heart Failure Association (HFA) of the ESC. Eur J Heart Fail 18(8):891-975

4. Nagueh SF, Smiseth OA, Appleton CP et al (2016) Recommendations for the evaluation of left ventricular diastolic function by echocardiography: an update from the American Society of Echocardiography and the European Association of Cardiovascular Imaging. J Am Soc Echocardiogr 29(4):277-314

5. Boyd AC, Richards DA, Marwick T et al (2011) Atrial strain rate is a sensitive measure of alterations in atrial phasic function in healthy ageing. Heart (British Cardiac Society) 97(18):1513-1519

6. Zhang Q, Yip GW, Yu CM (2008) Approaching regional left atrial function by tissue Doppler velocity and strain imaging. Europace 10(Suppl 3):iii62-iii69

7. Thomas L, Marwick TH, Popescu BA et al (2019) Left atrial structure and function, and left ventricular diastolic dysfunction: JACC state-of-the-art review. J Am Coll Cardiol 73(15):1961-1977

8. Singh A, Medvedofsky D, Mediratta A et al (2019) Peak left atrial strain as a single measure for the non-invasive assessment of left ventricular filling pressures. Int J Cardiovasc Imaging 35(1):23-32
9. Cameli M, Lisi M, Mondillo S et al (2010) Left atrial longitudinal strain by speckle tracking echocardiography correlates well with left ventricular filling pressures in patients with heart failure. Cardiovasc Ultrasound 8(14):1476-7120

10. Cameli M, Sparla S, Losito M et al (2016) Correlation of left atrial strain and Doppler measurements with invasive measurement of left ventricular end-diastolic pressure in patients stratified for different values of ejection fraction. Echocardiography 33(3):398-405

11. Kindermann M (2007) How to diagnose diastolic heart failure: a consensus statement on the diagnosis of heart failure with normal left ventricular ejection fraction by the Heart Failure and Echocardiography Associations of the European Society of Cardiology. Eur Heart J 28(21):2686 author reply-7

12. Dernellis JM, Stefanadis CI, Zacharoulis AA et al (1998) Left atrial mechanical adaptation to long-standing hemodynamic loads based on pressure-volume relations. Am J Cardiol 81(9):1138-1143

13. Peverill RE (2015) Left ventricular filling pressure(s): ambiguous and misleading terminology, best abandoned. Int $\mathrm{J}$ Cardiol 191:110-113

14. Nagueh SF, Smiseth OA, Appleton CP et al (2016) Recommendations for the evaluation of left ventricular diastolic function by echocardiography: an update from the American Society of Echocardiography and the European Association of Cardiovascular Imaging. Eur Heart J Cardiovasc Imaging 17(12):1321-1360

15. Kurt M, Tanboga IH, Aksakal E et al (2012) Relation of left ventricular end-diastolic pressure and $\mathrm{N}$-terminal pro-brain natriuretic peptide level with left atrial deformation parameters. Eur Heart J Cardiovasc Imaging 13(6):524-530

16. Ommen SR, Nishimura RA, Appleton CP et al (2000) Clinical utility of Doppler echocardiography and tissue Doppler imaging in the estimation of left ventricular filling pressures: a comparative simultaneous Doppler-catheterization study. Circulation 102(15):1788-1794

17. Mullens W, Borowski AG, Curtin RJ et al (2009) Tissue Doppler imaging in the estimation of intracardiac filling pressure in decompensated patients with advanced systolic heart failure. Circulation 119(1):62-70

18. Liu YY, Xie MX, Xu JF et al (2011) Evaluation of left atrial function in patients with coronary artery disease by twodimensional strain and strain rate imaging. Echocardiography 28(10):1095-1103

19. Mondillo S, Cameli M, Caputo ML et al (2011) Early detection of left atrial strain abnormalities by speckle-tracking in hypertensive and diabetic patients with normal left atrial size. J Am Soc Echocardiogr 24(8):898-908

20. Huynh QL, Kalam K, Iannaccone A et al (2015) Functional and anatomic responses of the left atrium to change in estimated left ventricular filling pressure. J Am Soc Echocardiogr 28(12):1428-1433

Publisher's Note Springer Nature remains neutral with regard to jurisdictional claims in published maps and institutional affiliations. 